Colorado Biorepository Core Facility and from the Plastic Surgery Clinics. Five micron sections from formalin-fixed paraffin-embedded samples were prepared for multiplex fluorescence immunohistochemistry by the Human Immunology \& Immunotherapy Initiative. We stained for CD20+, CD19+, and DAPI. Slides were imaged using Vectra $^{\circledR} 3$ scanning system from PerkinElmer. Images were analyzed in InForm ${ }^{\circledR}$ Tissue Finder, phenotpr, phenoptrReports by Akoya biosciences. RESULTS/ANTICIPATED RESULTS: We found a significant increase in the percentage of CD20 + and CD19+ B cells in keloid skin compared to normal skin tissue $(14.50 \%$ and $14.20 \%$ vs $6.47 \%$ and $7.56 \%$ of the total cells), respectively. Interestingly, we found that in the epidermis of keloid skin CD20+ cell were more abundant (14.46\%) whereas in the epidermis normal skin CD20+ cells were less predominant (5.14\%). In the dermis of keloid skin, CD20+ and $\mathrm{CD} 19+$ were in equal proportions $(13 \%)$ whereas in normal skin CD19+ cells were more predominant $(10.44 \%)$ compared to CD20+ cells (7.04\%). Dual positive B cells, CD19+/CD20+ cells, were more abundant in keloid dermis (11.06\%) compared to normal skin dermis (1.24\%). DISCUSSION/SIGNIFICANCE OF IMPACT: B cells are involved in fibroblast activation in diseases such as scleroderma and rheumatoid arthritis. With the increase of CD19+/CD20+ B cells in keloids, the role of $\mathrm{B}$ cells in keloid pathogenesis warrants further study. CD27 staining may determine if these are activated or follicular $B$ cells.

4353

\section{The Role of BCL2 Mediated Calcium Signaling on Leukemia Stem Cell Metabolism \\ Anagha Inguva ${ }^{1}$, Shanshan Pei ${ }^{2}$, Maria Amaya ${ }^{2}$, Brett Stevens ${ }^{2}$, Courtney Jones ${ }^{2}$, Daniel Pollyea ${ }^{2}$, and Craig Jordan ${ }^{2}$ \\ ${ }^{1}$ University of Colorado at Denver; ${ }^{2}$ University of Colorado}

OBJECTIVES/GOALS: The objective of this study is to define the molecular mechanisms that control survival of malignant stem cells in acute myeloid leukemia (AML). Leukemia stem cells (LSCs) are not effectively eradicated by standard treatment and lead to resistance and relapse, which contribute to poor survival rates. METHODS/STUDY POPULATION: The recently FDA approved venetoclax, a BCL2 inhibitor, with azacitidine, a hypomethylating agent leads to a $70 \%$ response rate in AML patients. Analysis of patients treated with this regimen showed direct targeting of LSCs. BCL2 has a non-canonical function in regulation of intracellular calcium. To determine how BCL2 mediated calcium signaling plays a role in LSC biology, we used LSCs isolated from venetoclax/ azacitidine (ven/aza) sensitive and resistant patient samples to measure expression of calcium channels via RNA seq. BIO-ID, siRNA, flow cytometry, seahorse assays, calcium measurements and colony assays were used to determine the effects of calcium channel perturbation on LSC biology. RESULTS/ANTICIPATED RESULTS: BCL2 inhibition leads to decreased OXPHOS activity in primary AML specimens. BIO-ID studies revealed cation/metal ion transporters, ER membrane proteins and ER membrane organization as top enriched pathways interacting with BCL2. RNA-seq data showed increased expression of genes involved in calcium influx into the ER in ven/aza sensitive LSCs and increased expression of genes involved in calcium efflux from the ER in ven/aza resistant samples. Ven/Aza resistant LSCs have increased mitochondrial calcium content, consistent with their increased OXPHOS activity as calcium is required for OXPHOS. Perturbation of these channels leads to decreased OXPHOS activity and decreased viability in LSCs.
DISCUSSION/SIGNIFICANCE OF IMPACT: We postulate that a deeper understanding of the mechanisms behind ven/aza targeting of LSCs will lead to the development of novel therapies for patients who do not respond to ven/aza. Our data show targeting intracellular calcium signaling could be a viable therapeutic strategy for AML patients.

The role of the L-type calcium channel, Cav1.3, in motor and associative learning

Aislinn Joanmarie Williams ${ }^{1}$, Marisol Lauffer, Hsiang Wen, and Bryn Myers

${ }^{1}$ University of lowa Institute for Clinical and Translational Science

OBJECTIVES/GOALS: Genetic variation in L-type voltage-gated calcium channels, including $\mathrm{Ca}_{\mathrm{V}} 1.3$, is associated with increased risk for psychiatric disorders including bipolar disorder and schizophrenia. Additionally, rare mutations in $\mathrm{Ca}_{\mathrm{V}} 1.3$ have been linked to epilepsy, developmental delay, and autism. Deletion of $\mathrm{Ca}_{\mathrm{V}} 1.3$ in mice is associated with impaired consolidation of contextual fear conditioning. Some studies have also observed affective behavior deficits in $\mathrm{Ca}_{\mathrm{V}}$ 1.3-deficient mice, but other studies have not found affective phenotypes, perhaps due to differences in genetic backgrounds, sex ratios, or task protocols. $\mathrm{Ca}_{\mathrm{V}} 1.3$ is important for slow afterhyperpolarization in hippocampal and amygdala neurons, which prevents excessive firing in response to sustained excitatory input, and $\mathrm{Ca}_{\mathrm{V}}$ 1.3-deficient amygdala neurons exhibit hyperexcitability and impaired LTP. $\mathrm{Ca}_{\mathrm{V}} 1.3$ is also expressed in the cerebellum, but its functional role there is not well understood. Given its importance in shaping neuronal activity in the hippocampus and amygdala, we hypothesized that loss of $\mathrm{Ca}_{\mathrm{V}} 1.3$ would cause abnormalities in motor learning as well as affective and cognitive behaviors. METHODS/STUDY POPULATION: Wild-type (WT), haploinsufficient (Hap), and knockout (KO) mice were maintained on a congenic $\mathrm{C} 57 \mathrm{BL} / 6 \mathrm{NTac}$ genetic background and were subjected to behavioral tasks including open field, rotarod, ErasmusLadder, elevated zero maze, forced swim test, and tail suspension test. Data were analyzed with sexes combined and with sexes separated to assess for sex as a biological variable. Studies were analyzed by one-way ANOVA, two-way ANOVA, or generalized linear mixed model, where appropriate. RESULTS/ANTICIPATED RESULTS: $\mathrm{Ca}_{\mathrm{V}} 1.3 \mathrm{KO}$ was associated with impaired motor learning in the rotarod task $(\mathrm{p}<0.05)$, as well as impaired associative learning in the ErasmusLadder task $(\mathrm{p}<0.01)$, despite intact locomotor function on both tasks. When examined by sex, the rotarod phenotypes were driven by motor learning impairments in males (both Hap and KO, $p<0.05$ and $p<0.01$, respectively), whereas the ErasmusLadder associative learning phenotypes were present in both sexes only in the $\mathrm{KO}$ condition, consistent with previously reported impairments in $\mathrm{Ca}_{\mathrm{V}}$ 1.3-deficient mice in consolidation of contextual fear conditioning. Although $\mathrm{KO}$ mice learned the motor aspects of the ErasmusLadder task, they learned more slowly. They also failed to learn start cues, which requires intact associative learning. No differences were observed in overall exploration or locomotor activity in open field or elevated zero maze. Analyses from affective tasks are ongoing. DISCUSSION/SIGNIFICANCE OF IMPACT: These preliminary studies provide new evidence that $\mathrm{Ca}_{\mathrm{V}} 1.3$ is important for the function of neural circuits involved in motor learning, and concur with previous data showing its involvement in associative learning. Our data differ slightly from previous studies of $\mathrm{Ca}_{\mathrm{V}} 1.3$ 
in motor learning, which could be attributable to differences in task protocols and/or genetic background. These results highlight the importance of $\mathrm{Ca}_{\mathrm{V}} 1.3$ in a variety of behaviors, which may help explain why variation in $\mathrm{Ca}_{\mathrm{V}} 1.3$ expression and function has pleiotropic effects in humans.

\section{7}

Transcriptome and molecular analysis of erythropoietininduced hypertension

Nitin Kumar ${ }^{1}$, Isabelle birt ${ }^{2}$, Kristina L. Hunker², and Santhi K.

Ganesh ${ }^{2}$

${ }^{1}$ University of Michigan School of Medicine; ${ }^{2}$ University of Michigan

OBJECTIVES/GOALS: High blood-pressure (BP) is a common adverse effect of erythropoietin (EPO) therapy among patients with chronic kidney disease on hemodialysis, and even among otherwise healthy individuals who receive EPO. In human genetics, EPO is associated with not only red blood cell traits, but hypertension (HTN) as well. Currently, there is no vascular gene expression data available in the setting of EPO-induced HTN that may explain precise role of key cellular players in its hypertensive etiology. Our aim is to characterize vascular transcriptome to identify key cellular players in EPOinduced HTN. METHODS/STUDY POPULATION: 10-12 week C57BL/6 male and female mice were randomly divided into two groups 1. Vehicle $(0.9 \%$ saline-VEH), 2. EPO, $(\mathrm{N}=4)$. VEH and EPO were intraperitoneal administered (EPO 75U/30g, 3 times/week) for 20 days. Blood-pressure was measured non-invasively via tail-cuff plethysmography. We characterized in-vivo transcriptome response of mouse descending aorta to EPO-HTN and vehicle control group by highthroughput RNA-sequencing. RESULTS/ANTICIPATED RESULTS: Systolic blood pressure (SBP) was significantly higher in EPO treatment, compared to vehicle (males and females combined SBP VEH 116.29 \pm 6.21 , EPO 129.57 \pm 4.59 , mean \pm s.d., adjusted $\mathrm{P}=0.0012$ ). Comparison of in-vivo transcriptional differences between vehicle and EPO-treated reveal statistically significant changes in cellular pathways consistent with hypertension such as upregulation of RAS signaling pathway and oxidative stress. In-vitro mouse aortic smooth muscle cells, EPO markedly increased phosphorylated-ERK activity, suggesting increased RAS activity. DISCUSSION/SIGNIFICANCE OF IMPACT: This study highlights the importance of previously unknown vascular key players and advances our understanding of the transcriptional events associated with EPO-induced hypertension.

Understanding the molecular mechanism of natural killer cell deficiency to improve natural killer cell in vitro differentiation for therapeutics

Megan Schmit ${ }^{1}$, Ryan Baxley ${ }^{1}$, Emily Mace ${ }^{2}$, Jordan Orange, Jeffery Miller $^{1}$, and Anja-Katrin Bielinsky ${ }^{1}$

${ }^{1}$ University of Minnesota; ${ }^{2}$ Columbia University Irving Medical Center

OBJECTIVES/GOALS: Natural killer (NK) cells are a potential cancer therapeutic but expanding NK cells efficiently in vitro is difficult. Natural killer cell deficiency (NKD), a primary immune deficiency affecting only NK cells, is caused by defects in several DNA replication proteins. By studying NKD we will achieve better NK cell in vitro differentiation. METHODS/STUDY POPULATION: One patient with NKD has a compound heterozygous mutation in the essential DNA replication protein MCM10. We hypothesize that in individuals with $\mathrm{NKD}$, dramatic telomere erosion from abnormal DNA replication leads to premature senescence and the loss of NK cells. To test our hypothesis, we will knockout one allele of MCM10 or over express MCM10 in NK cells isolated from blood. We will then monitor telomere length, expansion and cytotoxic activity of these NK cells. To understand the role of MCM10 in early stages of NK cell development we will deplete MCM10 in induced pluripotent stem cells and differentiate these cells into NK cells. During this differentiation we will monitor progression through NK cell developmental stages as well as telomere length and senescence markers. RESULTS/ANTICIPATED RESULTS: Telomeres insulate chromosomes and induce permanent growth arrest (senescence) when they are critically short. We have demonstrated that depletion of a DNA replication protein causes telomere erosion and increases senescence markers. NK cells have shorter telomeres and lower telomerase expression than other immune cells. We predict, this relatively poor telomere maintenance sensitizes NK cells to telomere loss upon depletion of replication proteins. During in vitro differentiation, we expect NK cell precursors to undergo premature senescence secondary to telomere shortening. Furthermore, we expect supplementation of DNA replication proteins will enhance NK cell expansion and maturation. DISCUSSION/SIGNIFICANCE OF IMPACT: NKD patients have provided the scientific community with clues as to what proteins NK cells rely on for their development. This project aims not only to understand why these proteins are critical, but to harness that information for cellular anti-cancer therapeutics.

4117

\section{UNIQUE VAGINAL MICROBIOME POPULATIONS AND MICROBIAL GENE CONTENT AMONG WOMEN WHO NATURALLY CONTROL HIV PROGRESSION}

Katherine Gisella Michel ${ }^{1}$, Bing Ma, Kathleen Weber, Leah McClellan, Anandi Sheth, Stephen Gange, Audrey French, Jacques Ravel, Igho Ofotokun, and Daniel Merenstein

${ }^{1}$ Georgetown - Howard Universities

OBJECTIVES/GOALS: The role of the vaginal microbiome (VM) in HIV disease progression is poorly understood. We examined VMs of $\mathrm{HIV}+$ Elite Controllers (ECs) and HIV+ Long-Term NonProgressors (LTNPs) compared to controls: HIV-positive antiretroviral (ARV) treated (HIV+ATs) and HIV-negative women in the Women's Interagency HIV Study (DC/Chicago/Atlanta sites). METHODS/STUDY POPULATION: VMs were surveyed via both $\mathrm{V} 3 / \mathrm{V} 4$ region of $16 \mathrm{~S}$ rRNA gene amplicon sequencing and metagenomics sequencing in 67 women across 4 study groups: 1) LTNPs (CD4 $>500$ cells/mL for $5+$ years without ARVs) $(\mathrm{n}=7)$ and 2$)$ ECs (HIV RNA $<80$ copies/mL for $2+$ years without ARVs) $(\mathrm{n}=8)$, matched with 3 ) HIV + ATs (on ARVs for $\geq 1$ year with CD4 increase $\geq 100$ cells $\left./ \mathrm{mm}^{3}\right) \quad(\mathrm{n}=34)$, and 4) HIV- women $(\mathrm{n}=18)$. Metagenomes were characterized from specimens collected at two time points: 1$)$ vaginal swabs collected 2016-2017 $(n=62)$ and 2) cervicovaginal lavage collected 2002-2016 $(\mathrm{n}=35$; DC/ Chicago only). We used VIRGO (human vaginal non-redundant gene catalog), a newly developed referencing framework to comprehensively catalog VM gene content, taxonomy and functions. RESULTS/ANTICIPATED RESULTS: Women were 89\% African American with a mean age of 46 years (SD 8.8). The most prevalent species were Gardnerella vaginalis (predominant in 34\%), 